\title{
Implicazioni medico-legali del trattamento endoscopico dell'ipertrofia prostatica benigna
}

\author{
- Aldo Carnevale ', Cristian D’Ovidio ${ }^{2}$ \\ Cattedra di Medicina Legale. Università “G. D’Annunzio”, Chieti \\ 2 Sezione di Medicina Legale. Università “G. D’Annunzio”, Chieti
}

\begin{abstract}
We report a case of chronic urinary and faecal incontinence after transurethral resection of the prostate (TURP) and inguinal erniotomy. The analisys of the surgical techniques used in the treatment of prostatic hypertrophy (IPB) and the characterization of the complications demonstrate that many adverse effects are due to faulty surgical technique. Diagnostic, prognostic and therapeutic aspects of prostatic hypertrophy are estimated and the "state of the art" of surgical treatment is traced, including positive and negative aspects. Particular attention was dedicated to the TURP, gold standard for IPB, singling out the risks and the possible iatrogenic injuries. Scientific references prove that the frequency of such injuries is wide distributed in endoscopic treatment and can be referred mainly to jatrogenic causes. The fundamental lines of professional liability are analyzed in order to the right of injury compensation, according to civil law.
\end{abstract}

Keywords: prostate hypertrophy, transurethral resection of the prostate, professional liability

Medical and legal aspects of endoscopic treatment of prostatic hypertrophy.

Pratica Medica \& Aspetti Legali 2008; 2(1): 9-20

\section{INTRODUZIONE}

Lipertrofia prostatica benigna (IPB) si avvale attualmente di svariati trattamenti farmacologici e chirurgici. Numerose, infatti, sono le metodiche chirurgiche utilizzabili, ciascuna di esse caratterizzata da vantaggi e svantaggi e gravata da rischi e sequele talvolta anche importanti. Tra queste, comunque, la TURP (Transurethral resection of the prostate) è la tecnica attualmente più utilizzata e considerata gold standard. Nel presente lavoro si ripercorrono brevemente le caratteristiche dell'ipertrofia prostatica benigna valutandone gli aspetti clinico-diagnostici e, in particolar modo terapeutici, soprattutto in relazione alle diverse tecniche chirurgiche prospettabili.

Particolare attenzione è stata dedicata alla TURP tentando di individuarne i rischi insiti nelle singole fasi operatorie, posto che l'occorrenza anche di importanti sequele dopo interventi sulla prostata, quali l'impotenza organica o l'incontinenza urinaria, possono essere frutto di responsabilità professionale con relative sequele risarcitorie.

Come noto, la funzione principale della prostata è quella di secernere, in associazione con le vescichette seminali, il liquido seminale, mediante il quale vengono convogliati gli spermatozoi all'interno della cavità vaginale; il liquido seminale, gli spermatozoi e le cellule desquamate dall'epitelio di rivestimento dei dotti escretori formano il liquido spermatico.

La ghiandola tende ad aumentare di volume sino alla pubertà per rimanere pressoché invariata sino alla quinta decade di vita; da tale epoca, in circa il $50 \%$ degli uomini [1] va incontro a ipertrofia e iperplasia, nella restante metà dei casi tende all'atrofia.

Fattori eziologici universalmente riconosciuti per l'ipertrofia prostatica benigna sono l'età e gli elevati livelli ematici di androgeni. 
L'iperplasia ha inizio in prossimità della zona di transizione, con primitivo incremento delle porzioni extranodulari e successivo interessamento di noduli di natura stromale e ghiandolare, fino alla formazione dei cosiddetti "lobi laterali". In sostanza, si verifica la proliferazione di noduli periuretrali in grado di ostacolare il flusso minzionale; alcuni studi [2] hanno, tuttavia, dimostrato l'assenza di correlazione tra dimensioni volumetriche della prostata ed esordio della sintomatologia ostruttiva urinaria, attribuendo un ruolo preponderante all'età della persona [3].

Da un punto di vista fisiopatologico, l'ostruzione al flusso sarebbe determinata dallo stiramento dei lobi prostatici iperplastici fino alla base vescicale, con conseguente deformazione e ostruzione del meato uretrale interno. Per tale ragione l'IPB dovrebbe essere ritenuto un processo iperplastico (non ipertrofico), con marcato aumento della popolazione cellulare [4].

La sintomatologia, nelle fasi iniziali della malattia, risulta dominata dalla riduzione della forza nel getto urinario [5]. Successivamente, sebbene tale condizione possa rimanere a lungo asintomatica, possono insorgere ritenzione completa acuta o cronica d'urina [6], infezioni ricorrenti e/o calcolosi urinaria, emospermia ed ematuria.

La diagnosi di IPB si basa innanzitutto sull'anamnesi, in grado di fare affiorare sintomi di natura irritativa (pollachiuria, gocciolamento minzionale, ecc.) od ostruttiva (difficoltà alla minzione con allungamento dei tempi del mitto, ecc.). L'esplorazione rettale consente di rilevare l'aumento di volume prostatico. Sebbene sia oramai universalmente riconosciuta l'insussistenza di una correlazione tra le dimensioni della ghiandola e indicazione all'intervento chirurgico, può rendersi ugualmente necessaria un'ecografia prostatica transrettale o sovrapubica transvescicale per individuare, oltre ad eventuali disomogeneità ghiandolari, le precise dimensioni prostatiche [7]. Un esame delle urine potrebbe, infine, permettere di identificare la contestuale presenza di ematuria o infezione delle vie urinarie. Di indubbio ausilio diagnostico risultano, inoltre, l'esame urografico (per difetti di riempimento del bassofondo vescicale), l'uroflussimetria, la valutazione del residuo post-minzionale e gli studi urodinamici di pressione-flusso. In alternativa, può effettuarsi l'uretrocistoscopia, da eseguire, eventualmente, in caso di preparazione all'intervento chirurgico [8].

\section{DESCRIZIONE DEL CASO}

Il caso in esame riguarda una persona di 60 anni che, a seguito di ipertrofia prostatica benigna e di ernia inguinale sinistra, veniva sottoposta, in unica seduta operatoria, a duplice intervento chirurgico.

Il soggetto, a seguito di stranguria e pollachiuria da adenoma prostatico, si era recato presso la Divisione di Urologia di un presidio ospedaliero, ove i Sanitari, dopo avere effettuato gli accertamenti diagnostici di routine, decidevano di intervenire con erniotomia inguinale sinistra e TURP. Il decorso post-operatorio fu indicato dagli urologi come sostanzialmente nella norma e conseguentemente si dispose la dimissione del paziente. Nel periodo successivo, tuttavia, il paziente, avendo accusato comparsa di incontinenza urinaria, si sottoponeva a cicli di elettrostimolazione della muscolatura perineale, con scarsi risultati. Persistendo la sintomatologia, lo stesso si ricoverò, per ulteriori 15 giorni, presso la Divisione Urologica di un altro ospedale. In tale sede, dopo ulteriori trattamenti e approfondimenti diagnostici, venne formulata diagnosi di urge e stress incontinence; una cistoscopia, inoltre, permetteva di rilevare un'apparente conservazione dello sfintere striato esterno e del veru montanum, oltre a una "rilevatezza" in loggia prostatica e a una piccola "guancia" apicale del lobo destro.

In un'ulteriore visita di controllo, oltre alla sostanziale persistenza della sintomatologia da incontinenza urinaria, gli urologi diagnosticavano ipotonia dello sfintere anale con indicazione all'applicazione di sfintere artificiale.

Da tale epoca il paziente è in condizioni stazionarie con cronica e irreversibile incontinenza urinaria e rilevante ipotonia dello sfintere anale.

Il contenzioso civilistico, iniziato per il risarcimento danni, si è da poco tempo concluso con addebito di responsabilità in capo ai chirurghi operatori e sostanziale soddisfacimento delle pretese risarcitorie avanzate dalla parte attrice.

\section{GLI ASPETTI CLINICI}

Il trattamento dell'IPB può essere effettuato sia per via farmacologica che chirurgica, utilizzando in tal caso un accesso retropubico o sovrapubico, ovvero endoscopico transuretrale.

Nonostante le diverse ipotesi terapeutiche, è certamente compito del medico indicare ai pazienti $\mathrm{i}$ rimedi igienico-dietetici per ridurre la congestione dei vasi pelvici e, nelle fasi irritative della malattia, può essere intrapresa terapia a base di: estrogeni, androgeni, progestinici, antagonisti della prolattina, mepartricina e alfa-bloccanti.

Quando la procedura chirurgica risulta inevitabile ed improcrastinabile, è comunque indispensabile una preliminare valutazione morfologica e funzio- 
nale del tratto urinario per individuare al meglio la procedura da adottare.

Lintervento chirurgico "a cielo aperto" sarebbe indicato nei pazienti con prostata solitamente di oltre $100 \mathrm{~g}$ di peso ovvero ove concomitino affezioni cardiovascolari, polmonari, diabetiche, neoplastiche, emocoagulative, ovvero quando vi sia calcolosi o diverticolosi vescicale. L'intervento chirurgico tradizionale sembra necessario anche nei casi in cui il paziente non possa essere oggettivamente sottoposto a intervento endoscopico (ad es. per grave artrosi dell'anca). Inoltre, in presenza di ernia inguinale, la prostatectomia potrebbe essere effettuata "a cielo aperto" sfruttando l'incisione mediana bassa utilizzata per la plastica erniaria peri-peritoneale [9].

La procedura chirurgica "a cielo aperto" viene distinta tra un accesso retropubico e uno sovrapubico; entrambi gli interventi, comunque, possono essere eseguiti in anestesia generale o peridurale. La prostatectomia retropubica consiste nell'enucleazione dell'adenoma prostatico attraverso un'incisione diretta della capsula prostatica anteriore. Quella sovrapubica, invece, porta all'enucleazione dell'adenoma attraverso un'incisione extraperitoneale della parete vescicale antero-inferiore. In ogni caso, è sempre necessario mantenere un tubo di drenaggio temporaneo e un catetere vescicale per alcuni giorni e la degenza ospedaliera, per tali procedure, non è inferiore a 6-8 giorni.

Laccesso retropubico avrebbe quale principale complicanza, sebbene morbilità e mortalità siano basse, l'abbondante emorragia per lesione del plesso venoso dorsale, distalmente all'apice della prostata; in tal caso, il chirurgo deve praticare subito una legatura dei peduncoli laterali della prostata a livello della giunzione prostato-vescicolare. In alcune circostanze può aversi incontinenza post-operatoria tendente a permanere settimane o addirittura mesi, per autolimitarsi sino a scomparire quasi definitivamente in seguito [10]. Soltanto in rarissime circostanze [11] comparirebbe un'incontinenza da sforzo, da lesione dello sfintere striato esterno per inadeguata capsulotomia trasversale ed enucleazione dell'adenoma. Le disfunzioni erettili si presentano nel 3-5\% dei casi [12], con maggiore diffusione nei soggetti anziani; soltanto il 2-3\% dei pazienti operati svilupperebbe sclerosi del collo vescicale a distanza di circa 612 settimane dall'intervento [12]. Tale complicanza deriverebbe dalla lesione dei nervi del plesso pelvico e dei nervi cavernosi in tre fasi dell'intervento: durante la dissezione dell'apice prostatico con sezione dell'uretra; in corso di mobilizzazione della prostata dal retto ed incisione della fascia pelvica laterale; durante la sezione dei peduncoli laterali.

La prostatectomia sovrapubica, adottata raramente per la scarsa visualizzazione del campo opera- torio e per la maggiore difficoltà all'enucleazione apicale, può essere causa di incontinenza urinaria post-operatoria ed emorragia intraoperatoria. Tale metodica risulta, peraltro, gravata da altre complicanze, quali: stravaso urinario, cistiti e orchiepididimiti dopo rimozione del catetere.

Anche nel caso di tale modalità chirurgica le complicanze erettili insorgerebbero soltanto nel 2-3\% dei casi [13], mentre l'eiaculazione retrograda (di entità variabile) si avrebbe nel $100 \%$ dei pazienti. L'eventuale insorgenza di incontinenza urinaria da urgenza, secondaria a instabilità vescicale, tenderebbe generalmente a risolversi nell'arco di 4-6 settimane.

In alternativa al trattamento chirurgico a cielo aperto, recentemente ha avuto grande diffusione la metodica endoscopica.

I primi sviluppi della TURP si ebbero negli anni '30, dopo l'invenzione della lampada incandescente da parte di Edison (1879), del cistoscopio di Nitze e Lieter (1887) e del tubo fenestrato di Hugh Hampton-Young. Nel 1932, infatti, Stearns utilizzò, per primo, un'ansa al tungsteno per resecare tessuto prostatico sotto visione diretta. Tuttavia, soltanto nei primi anni '70, con l'introduzione del sistema di illuminazione a fibre ottiche e con la scoperta della lente cilindrica, messa a punto da Hopkins, venne a svilupparsi, in maniera preponderante, la chirurgia endoscopica. Dopo la diffusione di tale metodica si susseguirono i rilievi statistici che documentarono l'efficacia dei risultati ottenuti con tale intervento. Nel 1989, Mebust e coll. individuavano nei sintomi d'ostruzione al deflusso vescicale in grado di interferire con la qualità della vita l'indicazione assoluta all'intervento. Successivamente, essi vennero sostituiti da: ritenzione urinaria acuta refrattaria a terapia, infezioni ricorrenti, ematuria ricorrente e marcata iperazotemia.

Allo stato attuale, si è concordi nel ritenere la TURP metodica gold standard per il trattamento dell'ipertrofia prostatica benigna in pazienti aventi prostata inferiore a 100 grammi di peso.

La TURP è eseguita in anestesia perdurale e ha una durata di circa 30-60 minuti; in corso di procedura, si effettua comunemente l'esame istologico del tessuto asportato con posizionamento finale di catetere vescicale, rimosso dopo pochi giorni; la degenza ospedaliera non supera di norma i 34 giorni.

Dopo la rimozione del catetere, i pazienti avrebbero un mitto non soddisfacente, per di più associato a sensazione di bruciore. La ripresa minzionale, sovente, si associa alla presenza di stimolo impellente di mingere che tende col tempo a scomparire. Tale urgenza minzionale temporanea sarebbe correlata al fatto che la vescica, solita ad aumentare la pressione interna per consentire il flusso urinario ostacolato dell'ipertrofia prostatica, continuerebbe a contrarsi a tali regimi ancora per un limitato periodo di tempo. 
Il controllo urologico dopo TURP si esegue ambulatorialmente con urinocoltura e uroflussimetria, oltre che, a cadenza annuale, con dosaggio del PSA. Da un punto di vista squisitamente tecnico-procedurale, la TURP ha inizio con la giusta calibrazione dell'uretra e prosegue introducendo in vescica 150 $\mathrm{ml}$ di soluzione non emolitica. La resezione dell'adenoma prostatico ha inizio a livello del collo vescicale, in posizione "ore 12 ", e continua verso il basso fino alla posizione "ore 9".

A seguito di resezione prostatica, si modifica il meccanismo dell'eiaculazione, venendosi a instaurare la cosiddetta eiaculazione retrograda o retrospermia. Lo sperma, anziché essere espulso all'esterno, refluisce in vescica e viene in seguito eliminato con le urine. Questa particolare modalità di eiaculazione è una conseguenza diretta ed inevitabile dell'asportazione chirurgica (insieme all'adenoma prostatico) delle fibre muscolari che chiudono il collo vescicale.

Alcuni Autori [14], tuttavia, avrebbero messo a punto una tecnica innovativa di TURP che, in caso di piccoli adenomi ostruttivi, consentirebbe di salvaguardare l'eiaculazione anterograda in più dell' $80 \%$ dei casi. La tecnica, studiata mediante ultrasonografia pre-eiaculatoria transrettale che avrebbe permesso di documentare l'assenza della fase di accumulo, consiste nel conservare una parte di tessuto prostatico sopra-montanale. La contrazione pre-eiaculatoria della parte di prostata risparmiata consentirebbe, infatti, di preservare l'eiaculazione anterograda.

Molta importanza è, comunque, rivestita dalla profondità di resezione, per cui è buona norma resecare fino al punto in cui le fibre muscolari circolari del collo vescicale risultano visibili. Un'eccessiva resezione del collo vescicale e una prolungata cauterizzazione potrebbero comportare la sclerosi del collo vescicale. L'intervento consiste nella resezione dell'adenoma per quadranti, mantenendo il resettore sempre innanzi al veru montanum, cercando di far terminare i lobi prostatici asportati all'interno della loggia mediana.

Altro punto critico dell'intervento è senza dubbio costituito dalla resezione della porzione posteriore del pavimento della loggia prostatica fino al veru montanum, poiché in tale fase può ledersi il trigono vescicale con conseguente incontinenza urinaria permanente; in ultimo, si procede alla resezione della porzione d'adenoma posta prossimalmente allo sfintere esterno, facendo, anche qui, molta attenzione a non procurare lesioni, onde evitare l'occorrenza di una stress incontinence.

In corso di TURP, possono essere provocate lesioni sfinteriali in tre zone diverse, differenziabili, a seconda della profondità, in lesioni a carico del veru montanum; dal veru montanum alla capsula prostatica; al di là della capsula prostatica.

Una lesione in seconda e terza porzione può determinare incontinenza urinaria significativa, in quanto l'intera regione periprostatica risulta attraversata da terminazioni dei nervi pudendo e pelvico, entrambi coinvolti nei processi della minzione e defecazione. Le terminazioni nervose efferenti del nervo pudendo interno sono localizzate nello sfintere striato esterno, in grado di contrarsi durante il riempimento vescicale e di rinforzare la minzione in assonanza con la contrazione del detrusore. Le terminazioni nervose del nervo pudendo esterno finiscono per interessare anche lo sfintere striato esterno del retto, in grado di inibire, per un periodo limitatamente lungo, il riflesso della defecazione. Le terminazioni del nervo pelvico, infine, finiscono per innervare il detrusore della vescica, lo sfintere liscio dell'uretra e, nel riflesso della defecazione, lo sfintere anale interno, tutti muscoli coinvolti nel controllo involontario della minzione e della defecazione.

Al termine della procedura endoscopica potrebbe permanere, in un numero molto variabile di casi (3,3-34,8\%) [15], una impotenza organica. Tale complicanza, secondo autorevoli studi [16], sarebbe dovuta a lesione iatrogena (da resettore) di nervi del plesso cavernoso che, in posizione "ore 5-7", in corrispondenza dell'apice ghiandolare, decorrono in assoluta contiguità con la capsula prostatica. La resezione del tessuto ghiandolare adenomatoso in tale zona dovrebbe, quindi, essere effettuata molto superficialmente, facendo attenzione a non ledere il plesso nervoso viciniore ${ }^{1}[17]$.

Nella Figura 1 vengono riportati i risultati di uno studio condotto su 629 pazienti, tra il 1999 e il 2004 , in cui è stato possibile riscontrare un incremento in percentuale pari al $12 \%$ di disfunzioni erettili dopo TURP, in soggetti con età media di $63,7 \pm 9,7$ anni [18].

Possono, dunque, ritenersi punti anatomici critici, a rischio di lesioni iatrogene in corso di TURP:

- lo sfintere uretrale interno;

- il trigono vescicale;

- lo sfintere uretrale esterno;

- la zona "ore 5-7" della capsula prostatica;

- le fibre muscolari del collo vescicale.

La TURP è comunque contraddistinta da indubbi vantaggi rispetto alle altre tecniche, quali:

- facilità d'esecuzione;

- buoni risultati funzionali;

- anestesia peridurale (non generale);

${ }^{1}$ I nervi della prostata traggono origine dal plesso ipogastrico e costituiscono un folto plesso, in cui sono presenti piccole formazioni gangliari. Dalle aree cerebrali originano impulsi nervosi che, attraverso le vie bulbo-pontine e spinali, raggiungono i centri toraco-lombare (T11-L2) e sacrale (S2S4). Dal primo originano i nervi ipogastrici (ortosimpatico), dal secondo i nervi pelvici (parasimpatico) che, fondendosi, formano i due plessi pelvici situati lateralmente alle vescichette seminali; dai due plessi pelvici originano i nervi cavernosi che si distribuiscono alle strutture erettili 
- tempo di degenza ospedaliera mai superiore ai 3-4 giorni;

- immobilizzazione post-operatoria minima;

- catetere vescicale per sole 24-36 ore;

- assenza di esiti cicatriziali;

- assenza di abbondanti emorragie postoperatorie e/o manifestazioni avverse legate all'anestesia generale;

- assenza di incontinenza urinaria, assenza di impotenza;

- assenza di alterazioni della sensibilità peniena. Anche nei soggetti ultraottantenni tale tecnica sembra fornire risultati accettabili in termini di morbilità e mortalità, con complicanze postoperatorie pari al 7,6\% e a sei mesi di distanza dall'intervento nel 6,39\% dei casi [19].

Negli ultimi anni si stanno diffondendo studi [20] su altre tecniche operative, come la rotoresezione transuretrale della prostata (TURRP), i cui risultati preliminari sembrerebbero molto simili a quelli della TURP, come evidenziato nella Figura 2.

La TUIP (Transurethral incision of the prostate) è eseguita con ansa di Collings incidendo la ghiandola dalle "ore 6" fino al veru montanum; la profondità di incisione è estesa fino al punto in cui divengono visibili i filamenti della capsula prostatica [21]. In corso d'intervento si effettua una biopsia della prostata per accertarsi di un'eventuale natura carcinomatosa del parenchima inciso. La metodica consiste nella disostruzione del collo vescicale per incisione endoscopica della massa prostatica ostruente; essa risulta particolarmente indicata in pazienti giovani e con dimensioni prostatiche abbastanza limitate. Tale tecnica, pur in mancanza di numerosi riferimenti bibliogra-

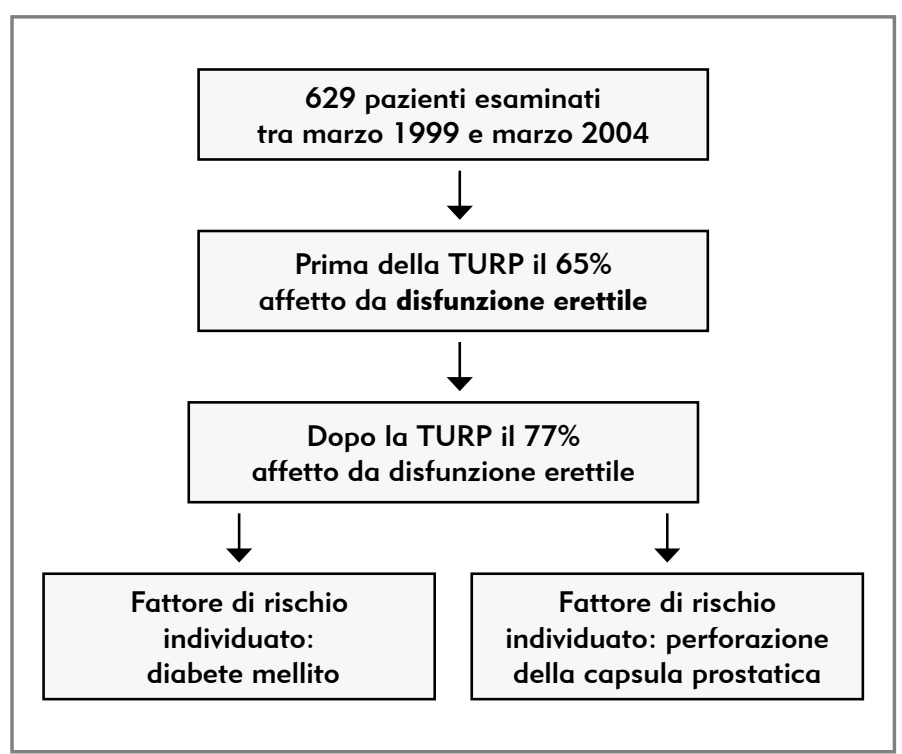

Figura 1. Incidenza di disfunzione erettile dopo TURP (transurethral resection of the prostate). Modificato da [18]

fici, sembra avere indubbi vantaggi rispetto alla TURP, rappresentati da: maggiore rapidità e facilità d'esecuzione; inferiore tasso di morbilità [22]; minore presenza di eiaculazione retrograda (655\%) [23]; rapida riduzione della sintomatologia nel 78-83\% dei casi [24]; impotenza nel 3,9-24,5\% dei casi [16].

La ILC (Interstitial laser coagulation), messa a punto da Hofstetter e Muschter, consiste nella coagulazione con fibra laser del tessuto prostatico adenomatoso. Il tubo sarebbe collegato ad un sistema a laserdiodi, con controllo a feed-back della temperatura. La tecnica laser, ancora non molto diffusa in campo urologico, presenta vantaggi [25] rispetto

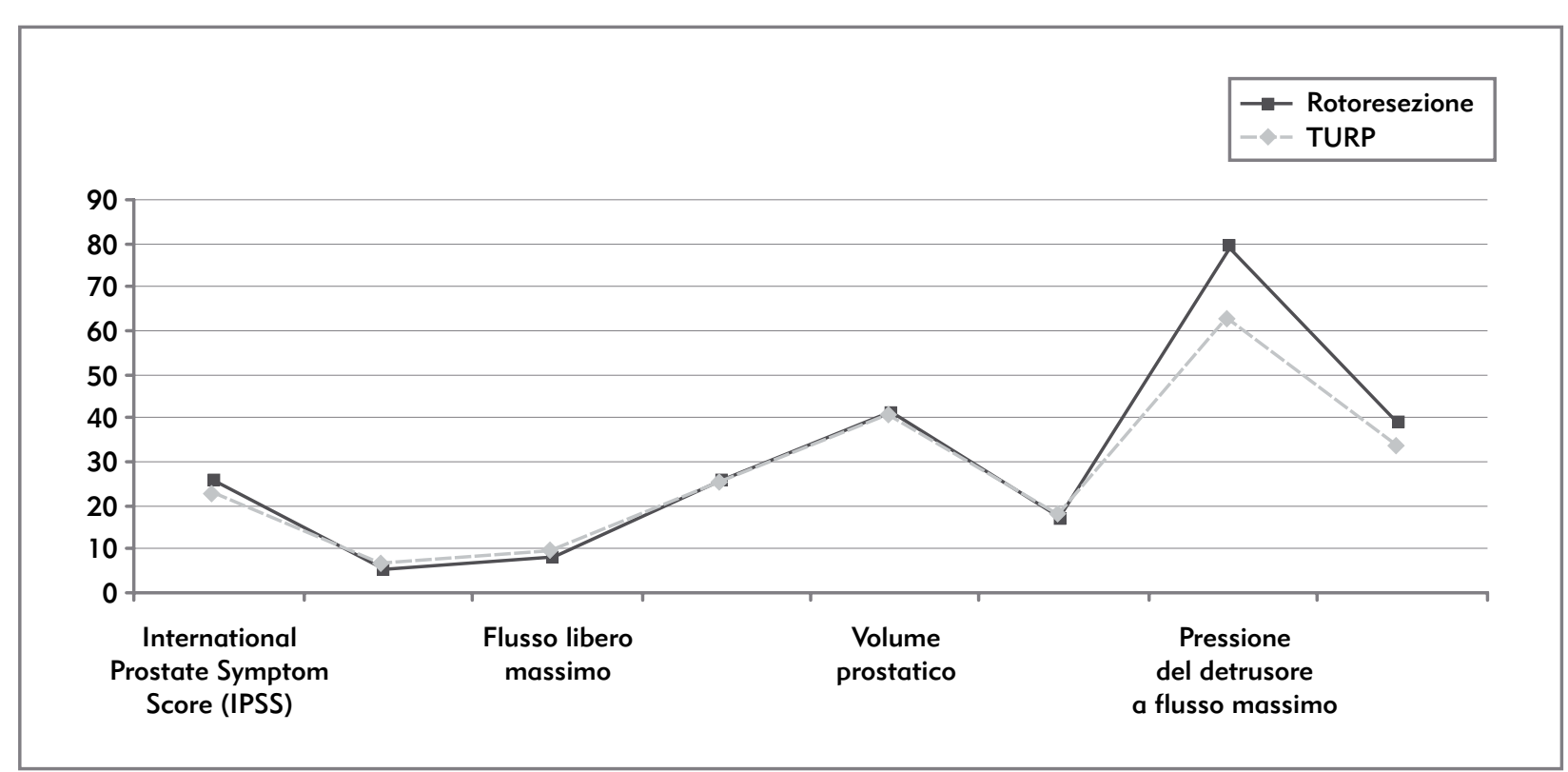

Figura 2. Confronto tra gli esiti di TURP (resezione trans uretrale della prostata) e TURRP (rotoresezione transuretrale della prostata). Modificata da [20] 
alle altre tecniche interventistiche, quali: minore pericolosità, minori quantitativi di fluido da irrigazione nel corso dell'intervento, degenza più breve, limitata ad un solo giorno, diminuzione del 63,3\% dell'AUA score (American Urological Association), diminuzione del $82 \%$ del residuo post-minzionale (RPM), diminuzione del 15\% del volume prostatico, aumento del $65 \%$ del flusso urinario $\left(Q_{\max }\right)$, sanguinamento post-operatorio pressoché assente [26], possibilità di trattare pazienti anche in terapia anticoagulante; ricorso possibile a un'anestesia locale o una sedazione endovenosa [27].

Vi sarebbero, tuttavia, alcuni svantaggi rispetto alla TURP [25]: l'innovatività della tecnica, non vi è tessuto disponibile per l'esame istologico, il costo elevato delle attrezzature, una maggiore dolorabilità post-operatoria, una maggiore possibilità di recidiva, la durata maggiore dell'intervento (circa 30' in più della TURP), il posizionamento di catetere per diversi giorni e una convalescenza più lunga. La sua scarsa invasività la renderebbe adatto per soggetti giovani, cui verrebbe risparmiata completamente la funzione sessuale e per pazienti molto anziani o ad alto rischio operatorio.

In uno studio [28] in cui vennero randomizzati 340 soggetti con IPB che furono sottoposti a TURP, a trattamento con laser neodimio-YAG ovvero a semplice osservazione clinica, dopo 7 mesi di follow-up, si riscontrò un buon risultato (in base a una scala di valutazione clinica) nell' $80 \%$ dei pazienti sottoposti a TURP, nel $64 \%$ di quelli trattati con laser e nel $18 \%$ del gruppo di osservazione. I pazienti trattati con laserterapia ebbero ricoveri ospedalieri più brevi, ma necessitarono di cateterismi vescicali più prolungati rispetto a quelli trattati per via endoscopica. Nessuna differenza significativa venne rilevata per quanto concerne le complicanze maggiori.

In un ulteriore studio [29], condotto su 82 pazienti sintomatici per IPB (ritenzione urinaria cronica e residuo post-minzionale di almeno $300 \mathrm{ml}$ ), trattati con TURP oppure laserterapia, dopo 7 mesi, si ottenne un buon risultato clinico-funzionale nell' $88 \%$ dei pazienti trattati chirurgicamente e nel $69 \%$ di quelli trattati con laser; in questo secondo studio, le complicanze maggiori (sanguinamenti e infezioni) furono significativamente più frequenti nel gruppo della TURP (30\% contro $8 \%$ ). In definitiva, sembrerebbe che la TURP, nonostante sia più efficace della laserterapia nel risolvere il quadro clinico dell'IPB, sarebbe gravata da una maggiore percentuale di complicanze.

La elettrovaporizzazione transuretrale della prostata venne intrapresa per la prima volta nel 1972 da Mebust e coll. al fine di assicurare una procedura rapida e con perdite ematiche minime in pazienti "ad alto rischio". Essa originariamente consisteva nell'utilizzo di una corrente di taglio di un bisturi elettrico in grado di surriscaldare ed essiccare la ghiandola prostatica. In tal modo, la por- zione adenomatosa della ghiandola andava incontro a desquamazione, con ripristino di un adeguato flusso urinario. Negli ultimi anni, tale tecnica è stata modificata con l'introduzione di un cilindro rotante in grado di vaporizzare il tessuto prostatico ostruente. Dopo avere misurato ecograficamente i diametri della prostata, si introduce per via transuretrale uno strumento al cui apice sono posizionati due aghi che vengono infissi nella prostata per scaricarvi energia; l'irrigazione con liquido sterile e il monitoraggio delle temperature intrauretrali riuscirebbero a evitare lesioni iatrogene. La procedura dura pochi minuti (20-30'), può essere eseguita in anestesia locale e in regime di day-hospital; viene posizionato un catetere vescicale per 24 ore e solitamente non si verificano episodi di ritenzione urinaria.

Dopo 30-60 giorni dall'intervento, si può apprezzare un notevole miglioramento del quadro clinico; la procedura è indicata nei pazienti che non rispondono adeguatamente alla terapia medica e nei quali si voglia evitare una resezione completa della ghiandola. I vantaggi di tale tecnica consistono in uno scarso sanguinamento peri e postoperatorio, nella mancanza di riassorbimento dei liquidi e in un ridotto periodo di cateterizzazione e degenza [30].

A seguito dei buoni risultati raggiunti, tale procedura avrebbe assunto un ruolo alternativo alla TURP e sarebbe indicata prevalentemente nel trattamento di pazienti aventi prostata adenomatosa di peso inferiore o uguale a $40 \mathrm{~g}$, con flusso massimo inferiore a $10 \mathrm{ml} / \mathrm{sec}$ o ritenzione completa e PSA inferiore a $4 \mu \mathrm{g} / \mathrm{l}$.

Le complicanze riscontrate con l'elettrovaporizzazione endoscopica della prostata [31] sarebbero rappresentate essenzialmente dalla persistenza di residui di tessuto adenomatoso e da stress incontinence temporanea nei 3 mesi successivi all'intervento, oltre a una maggiore incidenza di stenosi uretrali ${ }^{2}$ [32]. Un trial clinico randomizzato della durata di 5 anni ha evidenziato come tale tecnica operatoria sia, al pari della TURP, più indicata nelle ipertrofie prostatiche di media misura e risulti caratterizzata nel $17 \%$ dei casi da impotenza e nel $72 \%$ dei casi da eiaculazione retrograda [32].

Nei casi in cui, per l'età molto avanzata o per la gravità delle condizioni generali del paziente, si ritenesse comunque rischioso un intervento chirurgico, può ricorrersi, con un intervento effettuabile in regime ambulatoriale, al posizionamento di una protesi endouretrale (stent), costituita da un piccolo cilindro cavo spiraliforme di metallo che,

${ }^{2}$ Tale svantaggio sarebbe dovuto al fatto che il dispositivo utilizza un recettore e vaporizza solo $1 / 3$ della profondità di un'ansa da recettore per volta, per cui possono rendersi necessari più di tre passaggi (che provocano frizione sull'uretra) per ottenere la stessa cavità che si viene ad avere in una TURP di routine 
posto all'interno dell'uretra, la mantiene dilatata nel tratto in cui essa è ostruita da tessuto prostatico iperplastico.

Nella Figura 3 sono riportate le possibili sequele intraoperatorie, complessivamente risultate pari al 3,5\%, riscontrate su un'ampia casistica nei trattamenti endoscopici dell'ipertrofia prostatica benigna. Lo studio [33] è riferito a interventi di TURP $(92,6 \%)$, incisione prostatica transuretrale $(2,1 \%)$, elettrovaporizzazione transuretrale della prostata $(2,2 \%)$ e rotoresezione transuretrale della prostata $(1,1 \%)$.

Quelle spesso indicate in letteratura medico-scientifica come complicanze, sono in realtà sequele secondarie a difetti di manualità chirurgica o errori di tecnica da riferire a condotte inappropriate dell'operatore.

Certamente un esito inadeguato può conseguire ad un errore, ovvero alla reale sopravvenienza di una complicanza o per una reazione soggettiva anomala. Per poter discernere tra tali ipotesi, secondo il consueto canone di accertamento della colpa, l'indagine si incentrerà sulla prevedibilità e, in caso positivo, anche sulla evitabilità dell'evento non voluto. Ciò porta a ritenere che si possa escludere la ricorrenza di una responsabilità solo quando l'insorgenza di complicanze intraoperatorie siano del tutto impreviste e imprevedibili e di tale gravità, atipicità o complessità da sconvolgere il planning operatorio, turbare l'attenzione del chirurgo e dei suoi collaboratori e imporre prestazioni impegnative, inconsuete, impellenti o prolungate.

Bisogna, tuttavia, tenere ben distinte le complicazioni insite nella procedura dagli errori, ovvero dalle sequele derivanti da errori diagnosticoterapeutici.

I dati reperiti in letteratura sembrerebbero evidenziare come le sequele della TURP, in quanto comprese in un range molto ampio di probabilità, siano più che altro la conseguenza di errori derivanti non dalla tecnica in sé, quanto dall'esperienza e dalle capacità tecniche dei singoli operatori.

\section{GLI ASPETTI LEGALI}

Quanto sinteticamente affermato a chiusura del paragrafo precedente si desume dalla analisi della letteratura, eseguita nell'ambito di un caso direttamente osservato, sfociato poi in ambito giudiziario nel risarcimento del danno prodottosi a seguito di intervento di TURP.

Il caso riguardava un soggetto di sesso maschile che, affetto da anni da ipertrofia prostatica benigna, dopo un prolungato trattamento farmacolo-

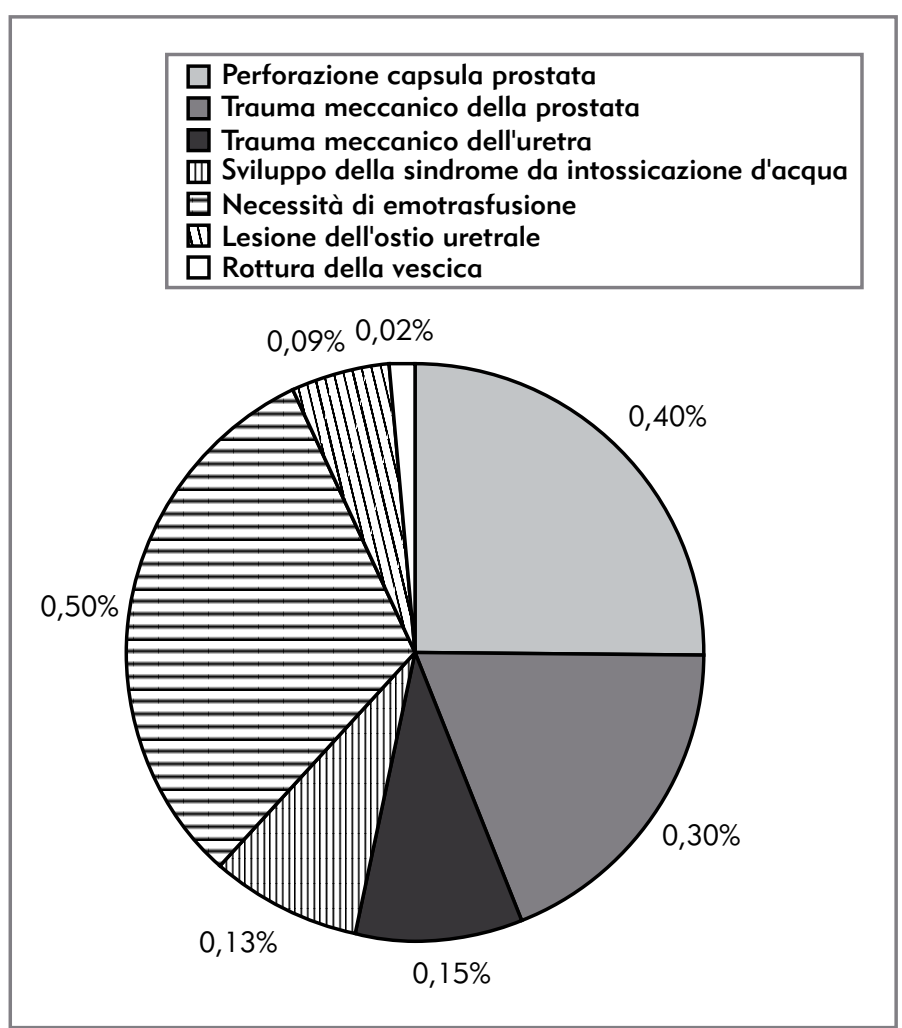

Figura 3. Sequele intraoperatorie degli interventi di TURP, incisione prostatica trans uretrale, elettrovaporizzazione transuretrale della prostata e rotoresezione transuretrale della prostata

gico, era indirizzato al trattamento chirurgico definitivo.

Stante la contestualità di un'ernia inguinale sinistra, gli urologi optavano per una soluzione chirurgica, consistita nella TURP e in una contestuale erniotomia inguinale sinistra.

Nonostante un iniziale decorso post-operatorio apparentemente nella norma, il paziente andava incontro ad incontinenza urinaria e fecale, la seconda da ipotonia dello sfintere anale, entrambe refrattarie ad elettrostimolazione perineale.

Lanalisi del caso ha comportato una rivisitazione degli eventi avversi connessi al trattamento chirurgico e/o endoscopico della ipertrofia prostatica benigna o delle possibili sequele, nell'ottica di una possibile ricorrenza di responsabilità professionale medica.

In generale si può affermare che addebiti di responsabilità possono ravvisarsi più in caso di inadeguato trattamento endoscopico che per omessa diagnosi di IPB o per adozione di metodica non appropriata. Tralasciando, quindi, possibili considerazioni sul misconoscimento della patologia, si è ritenuto opportuno focalizzare l'attenzione sui rischi connessi alla scelta del trattamento e, soprattutto, su quelli relativi alla fase esecutiva dell'intervento prescelto. Lindicazione al trattamento dovrebbe necessariamente scaturire da criteri (guidelines) previsti in letteratura medico-scientifica [34]; tali criteri presuppongono innanzitutto il discernimento tra ne- 
cessità d'intervenire per via chirurgica o proseguire con la terapia farmacologica. Una volta ritenuto necessario il trattamento chirurgico è indispensabile che il medico decida tra trattamento endoscopico ovvero "a cielo aperto": scelta questa di fondamentale importanza che deve tener conto anche dei rischi e delle sequele insiti nelle singole procedure quali ad esempio l'urgenza minzionale, l'incontinenza urinaria da sforzo o entrambe, la sclerosi del collo vescicale, l'eiaculazione retrograda e le disfunzioni erettili.

Ecco, dunque, che sulla base degli esami diagnostico-strumentali e delle opzioni terapeutiche a disposizione, lo specialista dovrebbe proporre al paziente l'ipotesi terapeutica più adeguata, modulata anche sui possibili rischi insiti nella specifica procedura. Peraltro, dovendosi considerare acquisite nel bagaglio culturale di ciascun urologo la conoscenza approfondita delle tecniche chirurgiche e/o soprattutto endoscopiche per il trattamento della IPB, si potrebbero ipotizzare profili di colpa in capo a quel professionista che optasse per la metodica più semplice, nonostante l'indicazione preferenziale ad altro approccio, in quanto gravata da minore complessità chirurgica.

A tale proposito è necessario ricordare che, a seguito di numerose pronunce giurisprudenziali, la figura del medico dipendente oggi è oramai accomunata, con vincolo di subordinazione, alla propria struttura d'appartenenza, assumendo lo stesso la qualifica, con quest'ultima, di debitore nei confronti del paziente.

Tale linea interpretativa, oggi diffusamente consolidata, trae origine dalla oramai nota sentenza della Cassazione civile del 22 gennaio 1999 n. 589, che assume l'obbligazione del medico dipendente, di natura contrattuale, ancorché non fondata su un contratto specifico, ma sul "contatto sociale". Ne consegue che attualmente i regimi di ripartizione dell'onere della prova, del grado della colpa e della prescrizione sono quelli tipici delle obbligazioni da contratto d'opera intellettuale.

In sostanza, con la sentenza n. 589 la Cassazione ha voluto ribadire il principio del diritto alla salute per affermare che, quand'anche non vi fosse contratto diretto tra medico ospedaliero e paziente, s'instaurerebbe ugualmente quel rapporto contrattuale, da semplice "contatto sociale", per cui rientrerebbe ugualmente nei doveri del medico, anche se di struttura pubblica, tutelare la salute così come stabilito dall'articolo 32 della Costituzione ${ }^{3}$.

Tale principio è stato recentemente ribadito dalla Suprema Corte nella sentenza n. 8826 del 13

\footnotetext{
${ }^{3}$ Volendo interpretare, in effetti, l'attuale tendenza giurisprudenziale seguita alla suddetta sentenza della Corte di Cassazione, deve ritenersi rilevante il contenuto del rapporto che inevitabilmente e obbligatoriamente s'instaura tra medico e paziente, non la fonte della stessa obbligazione
}

aprile 2007, nella quale, rifacendosi a un principio più svolte illustrato in sede di legittimità, la Corte ha sottolineato come l'accettazione del paziente in una struttura tesa all'assistenza sanitaria comporta di fatto la costituzione di un contratto definito "di prestazione d'opera atipico di spedalità". Tale principio troverebbe la sua ratio nel concetto secondo il quale la struttura sanitaria, costituzionalmente obbligata verso il cittadino a tutelarne il bene salute, si servirebbe per fare ciò proprio della prestazione professionale del medico.

Peraltro, per interventi di semplice esecuzione come la prostatectomia "a cielo aperto" nerve sparing, ovvero la TURP, sarebbe lecito attendersi risultati soddisfacenti, quantomeno a lungo termine [35]. Trattandosi, dunque, di interventi consueti e semplici, il paziente avrebbe l'onere di provare la "routinarietà" dell'atto chirurgico oltre al danno patito, mentre sull'urologo incomberebbe l'onere di provare che l'insuccesso sia dipeso da fatti non prevedibili, comunque indipendenti da proprie condotte. Diversa è l'interpretazione da fornire, invece, nei casi in cui i pazienti, per motivi diversi, debbano essere sottoposti a trattamenti di non frequente o facile esecuzione: in tali circostanze, infatti, potrebbe applicarsi quanto previsto dall'articolo 2236 c.c. (speciale difficoltà).

In tema di modalità d'accertamento della responsabilità professionale medica, di recente, si è assistito a un ulteriore cambiamento nell'orientamento della giurisprudenza. La Cassazione penale, infatti, con sentenza n. 4177 del 2 febbraio 2007, ha ridefinito, dopo quanto stabilito dalla celebre Sentenza "Franzese" (Cassazione penale, Sezioni Unite, n. 30328/2002), il valore da attribuire alle leggi statistiche in sede di accertamento del nesso causale per responsabilità medica omissiva. In passato per stabilire la sussistenza del nesso di causalità tra evento e condotta medica era in sostanza necessario un grado di probabilità prossimo alla certezza che l'azione doverosa, omessa dal Sanitario, avrebbe evitato l'evento lesivo, ovvero lo avrebbe fatto verificare in epoca significativamente posteriore e con minore intensità lesiva. Attualmente la Cassazione, in riferimento alle leggi statistiche, ha invece affermato che esse "sono solo uno degli elementi che il giudice può e deve considerare, unitamente a tutte le altre emergenze del caso concreto». La sentenza ha, peraltro, ribadito un criterio già ampiamente diffuso nell'accertamento del nesso di condizionamento, puntualizzando che «la dottrina e la giurisprudenza più convincenti sono unanimi nell'affermare che il giudizio sul nesso di causalità è giudizio da effettuarsi ex post, ad evento avvenuto e per il tramite del procedimento di eliminazione mentale [il cosiddetto "giudizio contro fattuale"], per cui, eliminata mentalmente la condotta, viene meno o non viene meno anche l'evento con certezza e con alta probabilità scientifica». 
Applicando tali nuovi orientamenti giurisprudenziali al caso concreto, per la verifica dell'esistenza, o meno, di un nesso causale tra condotta professionale, qualificata come censurabile, e il verificarsi del danno al paziente, si dovrebbero considerare, oltre alle modalità d'esecuzione dell'intervento, le diverse possibilità, in ossequio a leggi statistiche riconosciute da leggi scientifiche di copertura, di insorgenza di complicanze insite nella manovra.

D'altro canto, l'accertamento del nesso di condizionamento, in ambito penale e civile, segue regimi distinti, essendo molto più rigoroso in ambito penale, ove peraltro si distingue tra condotte omissive e commissive, rispetto a quello civile dove generalmente si segue il criterio dell'id quod plerumque accidit. In più, mentre in ambito civile l'onere della prova a discarico incombe sul debitore (il medico), in ambito penale l'onere è completamente a carico dell'accusa.

La sentenza di cui sopra (Cassazione penale, $n$. 4177 del 2 febbraio 2007), ribadisce e rafforza la validità dei criteri per l'accertamento del nesso di condizionamento usualmente utilizzati in ambito civile attribuendo estremo risalto alle leggi statistiche di copertura.

Per quanto attiene la TURP, procedura interventistica di più frequente esecuzione nei casi di IPB, ne sono stati identificati i rischi specifici per ciascuna fase della tecnica operatoria, giungendo alla conclusione secondo cui le regioni anatomiche maggiormente interessate da lesioni iatrogene in corso di procedura endoscopica sarebbero costituite dallo sfintere uretrale interno, il trigono vescicale, lo sfintere uretrale esterno, la zona "ore 5-7" della capsula prostatica e le fibre muscolari del collo vescicale.

In sostanza le moderne tecniche endoscopiche a disposizione dello specialista sarebbero oramai di uso comune, se ne conoscerebbero i risultati a breve e lungo termine e le possibili complicanze delle specifiche fasi operative. Per tali motivi è indispensabile che il chirurgo espleti la procedura con le modalità più idonee, attenendosi ai criteri operativi ormai evidenziati da gran parte delle Società Scientifiche Internazionali. È possibile, infatti, affermare che la condotta dello specialista, debba essere sempre valutata in riferimento a regole professionali, secondo quanto indicato dalla Cassazione (n. 4177 del 2 febbraio 2007), la cui inosservanza comporterebbe un addebito di responsabilità per l'operatore anche qualora le aspettative di successo dell'intervento fossero minime [36].

D'altronde, dalla casistica pubblicata è possibile desumere una chiave di lettura incontrovertibile: le sequele avrebbero una distribuzione molto variabile nelle singole tecniche endoscopiche, (TURP: 3,3-34,8\%; TUIP: 3,9-24,5\%; ecc.).

Tale dato, se confrontato con le dinamiche lesive precedentemente esaminate, farebbe ritenere le sequele in corso di procedura endoscopica frutto solo di inadeguata esperienza dell'operatore. In buona sostanza, un così ampio range d'insorgenza di una determinata "complicanza" non può che essere motivata dalla estrema variabilità nella manualità, esperienza e capacità del chirurgo.

Per tali ragioni, in caso di sequele quali quelle evidenziate nel caso in esame, si configurerebbe una "presunzione" di colpa in capo all'operatore. Principio, questo della "responsabilità presunta per legge", presente nel resto d'Europa ${ }^{4}$ e di fatto introdotta anche in Italia dalla recente sentenza della Suprema Corte n. 8826 del 13 aprile 2007.

In passato gran parte della dottrina riteneva che, quando venivano eseguiti di interventi chirurgici routinari ovvero di semplice esecuzione come molte delle procedure interventistiche esaminate per la IPB, dovesse pretendersi esclusivamente il corretto procedimento adottato per il raggiungimento del risultato. Oggi, invece, secondo quanto indicato nella citata sentenza (Cassazione Civile n. $8826 / 07$ ), viene decisamente accantonata la distinzione tra obbligazioni di mezzi e di risultato e si ribadisce che deve essere il medico a provare che il peggioramento (cui la Corte associa il non miglioramento) delle condizioni del paziente sia stato determinato da un evento imprevedibile e non superabile con l'adeguata diligenza.

Non meno importanti risultano altre due innovazioni apportate da tale pronunciamento, riassumibili nella caratterizzazione del concetto di diligenza del buon professionista e nell'onere di discarico da una presunzione di colpa anche in caso di mancato peggioramento del paziente.

Viene, infatti, stabilito che la diligenza richiesta al medico debba essere quella ordinaria del buon professionista, ovvero una diligenza particolarmente qualificata, che richiede la specifica conoscenza e applicazione delle cognizioni tecniche necessarie all'esecuzione di quella particolare attività professionale ( $\mathrm{Al}$ riguardo, in base al combinato disposto di cui agli artt. 1176, comma 2, e 2236 c.c. la diligenza richiesta è non già quella ordinaria del buon padre di famiglia [...] bensì quella ordinaria del buon professionista [...], e cioè la diligenza normalmente adeguata in ragione del tipo di attività e alle relative modalità di esecuzione [...]. Ai diversi gradi di specializzazione corrispondono infatti diversi gradi di perizia. Può allora distinguersi tra una diligenza professionale generica e una diligenza professionale variamente qualificata. Chi assume un'obbligazione della qualità di specialista, o una obbligazione che presuppone una tale qualità, è tenuto alla perizia che è normale della categoria»). Pertanto, secondo la Suprema Corte, la condotta del medico specialista deve es-

\footnotetext{
${ }^{4}$ In tal senso la proposta di direttiva del 9.11.90 del Consiglio delle Comunità Europee sulla responsabilità del prestatore di servizi (poi ritirata nel '94)
} 
sere esaminata con maggiore rigore in tema di responsabilità professionale, perché la qualifica professionale del debitore impone una diversa misura della perizia, a sua volta commisurata ai diversi gradi di specializzazione propri di quello specifico settore professionale.

Tale riferimento, profondamente innovativo, è contraddistinto dall'introduzione della dimostrazione che la Corte richiede al medico, che l'evento dannoso o il mancato miglioramento atteso si sia verificato per l'intervento di un fattore estraneo alla sfera soggettiva del medico e non sia ricollegabile causalmente al suo operato. In pratica, mentre in passato era necessario che il paziente (creditore nell'obbligazione contrattuale) dimostrasse oltre la routinarietà dell'intervento anche la modificazione peggiorativa indotta dal trattamento subito, allo stato attuale, secondo tale massima, si fa riferimento a una presunzione di colpa anche per il mancato miglioramento, solitamente atteso per quel determinato procedimento terapeutico.

Riassumendo, il nuovo orientamento giurisprudenziale in tema di accertamento della responsabilità medica in ambito civile si connota per un principio di generale favor per il danneggiato-creditore, con onus probandi sempre più rigoroso a carico del medico-debitore, su cui grava, di fatto, la prova del fatto impeditivo della prestazione nel caso di mancato raggiungimento del risultato, anche qualora non si fosse addivenuti a una modificazione peggiorativa dello status del paziente.

Peraltro, non può non considerarsi che l'attribuzione di responsabilità in capo all'urologo potrebbe spalancare scenari risarcitori sempre più vasti tra cui, in particolare, il così detto danno sessuale, risarcibile anche al partner del danneggiato.

In tema di danno alla capacità sessuale a carico del partner e al suo assoluto e acquisito diritto di ottenere un ristoro adeguato, non può non segnalarsi come la Corte di Cassazione [37], pronunciandosi in merito a complicanze neurologiche da prostatectomia radicale, abbia affermato: «Il comportamento doloso o colposo del terzo che cagiona ad una persona coniugata l'impossibilità dei rapporti sessuali è immediatamente e direttamente lesivo, sopprimendolo, del diritto dell'altro coniuge a tali rapporti, quale diritto-dovere reciproco, inerente alla persona, strutturante, insieme agli altri diritti-doveri reciproci, il rapporto di coniugio. La soppressione del diritto, menomando la persona del coniuge, nel suo modo di essere e nel suo svolgimento nella famiglia, è di per sé risarcibile, quale modo di riparazione della lesione di quel diritto della persona, qualificabile come danno che non è né patrimoniale (art. 2056 c.c. in relazione all'art. 1223 dello stesso codice) né non patrimoniale (art. 2059 c.c. in relazione al- l'art. 185 c.p.), comunque rientrante nella previsione dell'art. 2043 c.c.».

Si sostanzierebbe, dunque, a carico del coniuge sempre un danno ingiusto e si potrebbe intravedere anche un danno biologico (di natura psichica) se tale condizione comportasse un quadro clinico di natura psichiatrica. In sostanza la Cassazione [38] afferma che il danno arrecato al coniuge, per impossibilità ad avere rapporti sessuali col danneggiato, è senza dubbio risarcibile in quanto lesione di un diritto personalissimo: lo ius in corpus derivante dal vincolo civilmente garantito del matrimonio e tutelato ex articolo 2 della Costituzione.

\section{CONCLUSIONI}

Lipertrofia prostatica benigna (IPB) è dunque una patologia curabile e/o guaribile per via chirurgica. L'ampia incidenza di tale condizione e gli importanti risvolti civilistici che potrebbero emergere per il verificarsi di danni iatrogeni in corso d'intervento, costituiscono il principale motivo di preoccupazione per gli urologi.

La prima difficoltà per lo specialista è relativa alla scelta dell'opzione terapeutica più adeguata, posto che qualsiasi intervento è indubbiamente gravato da una certa quota di rischio. Da qui la necessità di informare adeguatamente il paziente anche al fine di concordare con esso l'intervento ritenuto più opportuno e la sua collocazione cronologica.

Dalla disamina della incidenza estremamente variabile delle sequele e complicanze che si possono osservare in corso di procedure endoscopiche per IPB si desume, peraltro, come gran parte degli eventi avversi siano determinati da una non perfetta esecuzione dell'atto chirurgico da parte dell'operatore. Ciò apre importanti scenari risarcitori che si estendono dallo specifico danno al paziente, a quello del coniuge nel caso di eventuale impotenza, secondo il principio dello ius in corpus posto in essere dal vincolo matrimoniale.

Tali ipotesi risarcitorie sono oggigiorno sempre più estese sia in rapporto all'attuale orientamento giurisprudenziale che considera la responsabilità del medico dipendente sempre di natura contrattuale sia per la profonda innovazione apportata dalla sentenza n. 8826 Cassazione civile che impone al medico una diligenza qualificata con conseguente spostamento dell'obbligazione assunta nei confronti del paziente verso il risultato atteso. 
1. Berry SJ, Coffey DS, Walsh PC, Ewing LL. The development of human benign prostatic hyperplasia with age.J Urol 1984; 132: 474-9

2. Sagnier PP, Macfarlane G., Richard F et al. Results of an epidemiological survey employing a modified American Urological Association Index for benign prostatic hyperplasia in France.J Urol 1994; 151: 1226-70

3. Jacobsen SJ, Girman CJ, Guess HA, Oesterling JE, Lieber MM.New diagnostic and treatment guidelines for benign prostatic hyperplasia. Potential impact in the United States. Arch Intern Med 1995; 155: 477-81

4. McNeal JG. The prostate gland: morphology and pathobiology. Urology 1983; 4: 3-33

5. Arrighi HM, Guess HA, Metter EJ et al. Symptoms and signs of prostatism as risk factors for prostatectomy. Prostate 1990, 16: 253

6. McConnell JD, Barry MD, Bruskewitz RC. et al. Benign prostatic hyperplasia: diagnosis and treatment. Clinical practice guideline. Agency for Health Care Policy and Research 1994; pub n. 94-0582

7. Barry SJ, Cockett ATK, Holtgrewe HL. Relationship of symptoms of prostatism to commonly used physiological and anatomical measures of the severity of benign prostatic hyperplasia. J Urol 1993; 150: 351-8

8. Abrams P. In support of pressure-flow studies for evaluating men with lower urinary symptoms. Urology 1994; 44: 153-5

9. Schlegel PN, Walsh PC. Simultaneous preperitoreal hernia repair during radical pelvic surgery. J Urol 1987; $137: 1180$

10. Moinzadeh A, Shunaigat AN, Libertino JA. Urinary incontinence after radical retripubic prostatectomy: the outcome of a surgical technique. BIU Int 2003; 92: 355-9

11. Mebust WK, Holtgrewe HL, Cockett ATK. Transurethral prostatectomy: immediate and postoperative complications. A cooperative study of 13 participating institutions evaluating 3885 patients. J Urol 1989; 141: 243

12. Reiner WG, Walsh PC. An anatomical approach to the surgical management of the dorsal vein and Santorini's plexus during radical retropubic surgery.J Urol 1979

13. Wakabashi Y, Kim CJ, KataokanA. Urinary continence after radical retropubic prostatectomy: a modification in the technique of apical dissection. Hinyokika Kiyo 2003; 49: 503-7

14. Malament M. Maximal hemostasis in suprapubic prostatectomy. Surg Gynecol Obstet 1965; 120: 1307

15. Ronzoni G, De Vecchis M. Preservation of anterograde ejaculation after transurethral resection of the prostate (TURP) and the bladder neck (TURBN), Università Cattolica del Sacro Cuore - Roma, 1997

16. Barry MJ, Fowler FJ, O'Leary MP. et al. The American Urological Association's symptom index for benign prostatic hyperplasia. J Urol 1992; 148: 1549-57

17. Grasso M. e coll., Reevaluation of impotence following TURP. Minerva Urol Nefrol 1993; 45: 105-8

18. Poulakis V, Ferakis N, Witzsch U, de Vries R, Becht E. Erectile dysfunction after transurethral prostatectomy for lower urinary tract symptoms: results from a center with over 500 patients. Asian J Androl 2006; 8: 69-74

19. Chtourou M, Binous MY, Kbaier I, Hafsia G, Kallel Y, Horchani A, Endoscopic resection of benign prostatic hyperplasia in men over 80 years of age: a retrospective study of 184 cases. Tunis Med 2004; 82: 725-9

20. Soliman SA, Wadie BS, Ibrahim el-HE, Shehab El-Dein AB, Rotoresection versus transurethral resection of the prostate: shortterm evaluation of a prospective randomized study. J Urol 2007; 177: 1036-9

21. Edwards LE, Bucknall TE, Pittam MR. Transurethral resection of the prostate and bladder neck incision: a review of 700 cases. BrJ Urol 1985; 57: 168-71

22. Orandi A. Transurethral incision of the prostate. J Urol 1973, 110: 229-31

23. Dørflinger T, Jensen FS, Krarup T, Walter S. Transurethral prostatectomy compared with incision of the prostate in the treatment of prostatism caused by small benign prostate glands. Scand J Urol Nephrol 1992; 148: 1088-90

24. McConnell JD. e coll., Benign prostatic Hyperplasia: diagnosis and treatment. Clinical Practice Guideline., No. 8, AHCPR Publication. Rockville, MD, Agency for Health Care Policy and Research Public Health Services, US Department of Health and human services, 1994

25. Tasso M, Formiconi A, Pop F, Sedigh O, Ferrando U. Coagulazione laser interstiziale (ILC) nell'ipertrofia prostatica benigna. Torino: $1^{\circ}$ Congresso Nazionale Società Urologia Nuova, 28-30 ottobre 1999

26. Kingston TE, Nonnenmacher AK, Crowe H, Costello AJ, Street A. Further evaluation of transurethral laser ablation of the prostate in patients treated with anticoagulant therapy. Aust N Z J Surg 1995; 65: 40-3

27. Leach GE. Local anesthesia for laser prostatectomy. J Endourol 1995; 5: 159-61

28. Muschter R. et al. One year experience in interstitial laser coagulation for benign prostatic hyperplasia.J Urol 1993; $149: 1012$

29. Aslam M, Lynch MD, O’Donoghue N. Interstitial laser coagulation for the treatment of urinary outflow obstruction.J Urol 1993; 149: 1

30. Corbu C, Traverso P, Bonini F, Simonato A, Farneti F, Carmignani G. Elettrovaporizzazione transuretrale della prostata: risultati a un anno di distanza. Istituto di Clinica Urologica "L.Giuliani”. Università di Genova, $46^{\circ}$ Convegno SUNI: Reggio Emilia, 23-25 Ottobre 1997

31. Berti GL, Leidi GL, Raimoldi A, Veneroni L, Giola V, Longoni E et al. Elettrovaporizzazione endoscopica della prostata (TVP): esperienza su 112 casi, U.O. di Urologia. Ospedale di Legnano, 1997 
32. Hammadeh MY, Madaan S, Hines J, Philp T. 5-year outcome of a prospective randomized trial to compare transurethral electrovaporization of the prostate and standard transurethral resection. Urology 2003; 61: 1166-71

33. Martov AG, Kornienko SI, Gushchin BL, Ergakov DV, Sazonov OA. Intraoperative urological complications in transurethral surgical interventions on the prostate for benign hyperplasia. Urology 2005; 4: 3-8

34. Roehrborn CG, McConnell JD, Barry MJ, Benaim E, Bruskewitz RC, Blute ML et al. AUA Guidelines on management of benign prostatic hyperplasia. American Urological Association Education and Research, 2003

35. Mulhall JP, Slovick R, Hotaling J, Aviv N, Valenzuela R, Waters WB et al. Erectile dysfunction after radical prostatectomy: hemodynamic profiles and their correlation with the recovery of erectile function. J Urol 2002; 167: 1371-5

36. Cassazione, sentenza n. 6141 del 21 dicembre 1978. Giurisprudenza Italiana 1979; 1: 953

37. Cassazione, sentenza n. 4671 del 21 maggio 1996.

38. Cassazione civile, sez. III, sentenza n. 6607 del 11 novembre 1996

\section{Ulteriori approfondimenti}

- Avecone P. La responsabilità penale del medico. Padova: Vallardi, 1981

- Barni M. Impunità e colpa: il concetto di responsabilità professionale alla luce dell'evoluzione sociale e giuridica. Riv Ital Med Leg 1996; 18: 353-64

- Bilancetti M. La responsabilità penale e civile del medico. Padova: Cedam, 1998

- Canzio G. La responsabilità per colpa professionale nell'ambito del SSN. Arch Med Leg Ass 1994; 16: 103-13

- Carusi D. Responsabilità del medico, prestazione professionale di speciale difficoltà e danno alla persona. Giurispru Ital; 143: 559-604

- Conti A. Errore medico e dovere di informarne il paziente. Riv Ital Med Leg 1998; 20: 1171-9

- Fiandaca G. Il reato commissivo mediante omissione. Milano: Giuffrè, 1979

- Fineschi V. Res ipsa loquitur: un principio in divenire nella definizione della responsabilità medica. Riv Ital Med Leg 1989; 11: 419-54,

- Fiori A. La Medicina difensiva. Riv Ital Med Leg 1996; 899

- Fiori A. Medicina legale della responsabilità medica. Milano: Giuffrè, 2000

- Gerin C. La valutazione medico legale del danno alla persona e responsabilità civile. Milano: Giuffrè, 1987

- Giannini G. Onere della prova e responsabilità professionale medica. Resp Civ Prev 1992; 27: 177-84

- Ingrassia G. La responsabilità del medico per fatti colposi dipendenti dalla sua professione. Criminol Psicopatol Forense 1994; 10: $107-9$

- Mazza L. Il nesso di causalità nella responsabilità professionale. Riv Ital Med Leg 1994; 16: 385-405 